\title{
Preparation and Microwave Absorbing Properties of Hollow Sphere $\mathrm{Fe}_{3} \mathrm{O}_{4} / \mathrm{Co}$ Composite
}

\author{
Yanhong Fu*, Guimei Shi, Zhijie Li \\ School of Science, Shenyang University of Technology, Shenyang, China \\ Email address: \\ fyhvip@126.com (Yanhong Fu), gmshi@imr.ac.cn (Guimei Shi), zjli@imr.ac.cn (Zhijie Li)
}

\section{To cite this article:}

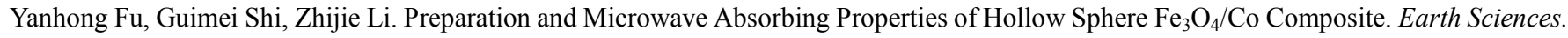
Vol. 4, No. 6, 2015, pp. 247-251. doi: 10.11648/j.earth.20150406.15

\begin{abstract}
In this paper, the hollow sphere $\mathrm{Fe}_{3} \mathrm{O}_{4}$ powders pepared by the solvothermal method were mixed with Co nanoparticles (prepared by arc-discharge, the size about $100 \mathrm{~nm}$ ), the quality ratio of $\mathrm{Fe}_{3} \mathrm{O}_{4}$ and Co is $4: 1$. The SEM study shows that the $\mathrm{Fe}_{3} \mathrm{O}_{4}$ powders have Hollow sphere structural with the size of about 200nm. As a result, when Co nanoparticles are introduced, $\mathrm{Fe}_{3} \mathrm{O}_{4} / \mathrm{Co}$ composite absorbent is obtained enhanced microwave absorption properties, which is attributed to the improved synergistic effect of dielectric and magnetic components between $\varepsilon_{r}$ and $\mu_{r}$. The results show that $\mathrm{Fe}_{3} \mathrm{O}_{4} / \mathrm{Co}$ composite absorbent is great potential microwave absorbents for practical applications.
\end{abstract}

Keywords: Hollow Sphere $\mathrm{Fe}_{3} \mathrm{O}_{4}$, Co, Composite, Absorbing Properties

\section{空心 $\mathrm{Fe}_{3} \mathrm{O}_{4} / \mathrm{Co}_{0}$ 纳米复合材料的制备及吸波性能研究}

\section{付艳宏，史桂梅，李志杰}

理学院, 沈阳工业大学, 沈阳, 中国

\section{邮箱}

fyhvip@126. com(付艳宏), gmshi@imr.ac. cn(史桂梅), zj1i@imr.ac.cn(李志杰)

摘要: 本文将制备的空球四氧化三铁 $\left(\mathrm{Fe}_{3} \mathrm{O}_{4}\right)$ 与Co纳米粉 (直流电弧法制备, 尺寸大约为 $\left.100 \mathrm{~nm}\right)$ 混合制备 $\mathrm{Fe} \mathrm{O}_{3} / \mathrm{Co}_{4}(4: 1)$ 微纳米复合微波吸收剂。研究结果表明, 利用水热法合成的 $\mathrm{Fe}_{3} \mathrm{O}_{4}$ 为空球结构, 尺寸大小约为 $200 \mathrm{~nm}$ 。通过实验研究表 明, $\mathrm{Co}$ 纳米粉的加入, 有效地调节了 $\mathrm{Fe}_{3} \mathrm{O}_{4}$ 吸收剂介电损耗和磁损耗的协同作用，大大提高了其吸波性能，因此 $\mathrm{Fe}_{3} \mathrm{O}_{4} / \mathrm{Co}$ 是一种具有潜在实际应用价值的微波吸收剂。

关键词：空心四氧化三铁, 钴, 复合材料, 吸波性能

\section{1. 引言}

随着无线电技术的迅猛发展, 电子产品及设备在为人 们的工作生活和社会建设提供了便利与贡献的同时, 这些 电子产品及设备在工作运转时所产生的电磁辐射和电磁 波干扰不仅影响到了我们正常的通讯, 而且也造成了人类 生存空间中环境的日益恶化, 影响了我们的生活质量, 越 来越多的临床实例证明电磁污染已经威胁到了人类的健
康。电磁波吸收材料是一种能够将外界入射的电磁波吸收、 衰减并转化成热能或其他形式的能量的一类功能材料, 目 前, 高性能的吸收电磁波的材料已经成为各国重点研究项 目之一。吸收材料不仅可以降低电磁波对环境的影响, 减 少对人类健康的损害, 而且能衰减或消除雷达等的探测信 号。吸波材料不仅作为现代飞行器的基础材料, 更是军事 隐身技术的重要研究热点, 目前吸波材料的研究和应用大 部分都是针对雷达波段 [1-3]。综上所述, 研究吸波材料 
不论是在人类生产生活中还是在军事装备力量上都具有 深远意义 $[4]$ 。

传统的吸波材料中，铁氧体系吸波材料因为自然资 源丰富、频率特性良好、制备工艺简单而突出。铁氧体 材料是一种既有复磁导率又有复介电常数的双复介质, 其中对电磁波的介电损耗主要来自极化效应, 而在对电 磁波的磁损耗方面, 主要是由自然共振决定, 所以铁氧 体吸波材料可以同时产生磁损耗和介电损耗 [5-8]。从20 世纪 50 年代开始铁氧体系吸波材料已被广泛使用, 而 $\mathrm{Fe}_{3} \mathrm{O}_{4}$ 作为典型的铁氧体, 由于其具有特殊的电学磁学的 特性而备受各国科学家们的关注, $\mathrm{Fe}_{3} \mathrm{O}_{4}$ 材料在电磁波吸 收剂领域占有着重要的地位, 是一种极具发展前景的吸 波材料 $[9,10]$ 。近年来, Zhang [11]等采用共沉淀法制备 了油酸 $(\mathrm{UA})$ 改性的 $\mathrm{Fe}_{3} \mathrm{O}_{4} @ \mathrm{UA}$, 由于分散剂油酸分子在 $\mathrm{Fe}_{3} \mathrm{O}_{4}$ 表面上形成长链烷烃层, 所形成的纳米粒子不粘连, 稳 定分散。 $\mathrm{Lu}[12]$ 等利用微乳液法在始终通Ar气状态下, 以正庚烷作油相, 制备了不同粒径的 $\mathrm{Fe}_{3} \mathrm{O}_{4}$ 磁性纳米粒子。 Lemine [13] 等利用改进的溶胶凝胶法, 得到 $\mathrm{Fe}_{3} \mathrm{O}_{4}$ 磁性纳 米颗粒。但是, $\mathrm{Fe}_{3} \mathrm{O}_{4}$ 吸波材料本身密度较大、质量重, 使其在电磁波吸收应用中受到一定限制, 难以同时达到 理想吸波材料的要求 [14], 只有通过细化和复合来改善 电磁参数, 才能有效提高吸波性能。空心 $\mathrm{Fe}_{3} \mathrm{O}_{4}$ 纳米材料 不仅质量轻 [15], 而且比表面积大, 可以造成多重散射, 而且表面较多的悬挂键可以导致界面极化的增强, 这些 散射和极化可以增加材料对电磁波的吸收和衰减, 从而 改善材料的吸波性能。所以, 本研究设计合成空心 $\mathrm{Fe}_{3} \mathrm{O}_{4}$ 与其它物质复合的吸波材料有着重要的意义。通常Co也 是一种吸波剂, 但主要在高频段中有较强的吸收 [16], 为了改善 $\mathrm{Fe}_{3} \mathrm{O}_{4}$ 的吸波性能, 本文将制备的空球 $\mathrm{Fe}_{3} \mathrm{O}_{4}$ 与 $\mathrm{Co}$ 纳米粒子复合, 以寻找性能优良的微波吸收剂。

\section{2. 实验部分}

\section{1 . 实验原料}

本实验采用的原料均采购于国药集团化学试剂有限 公司, $\mathrm{Co}_{0}$ 纳米粉, 聚乙烯吡咯烷酮 $\left(\left(_{6} \mathrm{C}_{9} \mathrm{NO}\right)_{\mathrm{n}}\right)$ 优级纯和氯 化高铁 $\left(\mathrm{FeCl}_{3} \cdot 6 \mathrm{H}_{2} \mathrm{O}\right)$ 、六次甲基四胺 $\left(\mathrm{C}_{6} \mathrm{H}_{12} \mathrm{~N}_{4}\right)$ 、无水乙醇 $\left(\mathrm{C}_{2} \mathrm{H}_{6} \mathrm{O}\right)$ 、乙二醇 $\left(\mathrm{C}_{2} \mathrm{H}_{6} \mathrm{O}_{2}\right)$ 的分析纯。

\section{2. 样品制备及测试}

实验采用水热法制备空心 $\mathrm{Fe}_{3} \mathrm{O}_{4}$, 将 $0.675 \mathrm{~g}$ 氯化高铁 $\left(\mathrm{FeCl}_{3} \bullet 6 \mathrm{H}_{2} \mathrm{O}\right) ， 0.14 \mathrm{~g}$ 六次甲基四胺（HMT），0.25g聚乙 烯吡咯烷酮 (PVP), 溶于 $15 \mathrm{~mL}$ 乙二醇 (EG) 中, 磁力搅拌, 使之形成黄色透明溶液。然后将该溶液转移入容积适合的 高压反应釜中。密封之后, 将反应釜放入电热鼓风干燥箱 中, $200^{\circ} \mathrm{C}$ 保温 12 小时, 反应结束后自然冷却到室温。再 将所得的黑色粉末进行离心分离, 用无水乙醇和蒸馏水清 洗多次, $60^{\circ} \mathrm{C}$ 真空干燥 6 小时后收集。将得到的空球 $\mathrm{Fe}_{3} \mathrm{O}_{4}$ 与直流电弧法制备的 $C_{0}$ 纳米粉进行质量比为 $4: 1$ 的混合, 吸波剂与石蜡质量比为 $7: 3$ 的混合做成待测吸波样品。样 品的相结构、形貌分别用X-Ray衍射 (BD2000 XRD Cu K $\alpha$ $\lambda=0.15405 \mathrm{~nm})$ 、扫描电镜 $(\mathrm{JSM}-635 \mathrm{~F}, \mathrm{Jeo}$ ) 确定, 其吸波性能用ENA5071C矢量网络分析仪完成。

\section{3. 结果与讨论}



图1 空心球 $\mathrm{Fe}_{3} \mathrm{O}_{4}$ 的X-Ray衍射图谱。

图1. 是所制备样品我们通过XRD来验证了所合成的 $\mathrm{Fe}_{3} \mathrm{O}_{4}$ 空心球的相结构。从图中可以清晰地看到, 在 $2 \theta$ $=30.1 、 35.4 、 37.1 、 43.1 、 53.4 、 57.0 、 62.6^{\circ}$ 出现的 衍射峰, 分别对应 (220)、(311)、(222)、(400)、(422)、 (511)、(440) 晶面。说明本研究所合成的空心球材料为面 心立方相的 $\mathrm{Fe}_{3} \mathrm{O}_{4}$ 的标准衍射卡片相吻合 (JCPDS card No. 19-0629)。
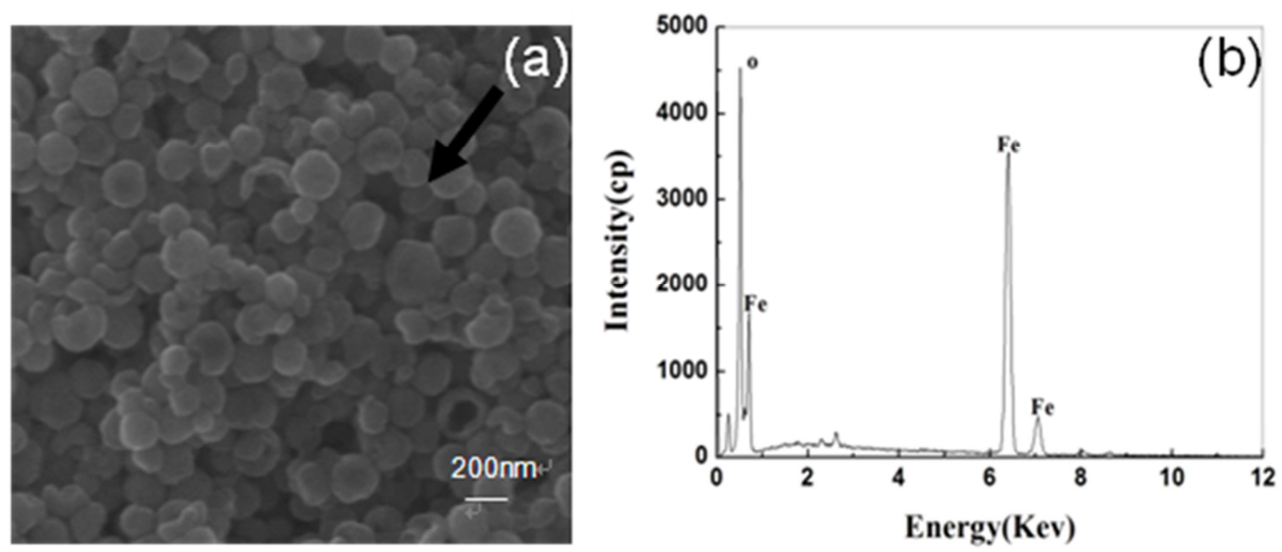

图2 空心球 $\mathrm{Fe}_{3} \mathrm{O}_{4}$ 的扫描电镜（SEM）照片与能谱图。 


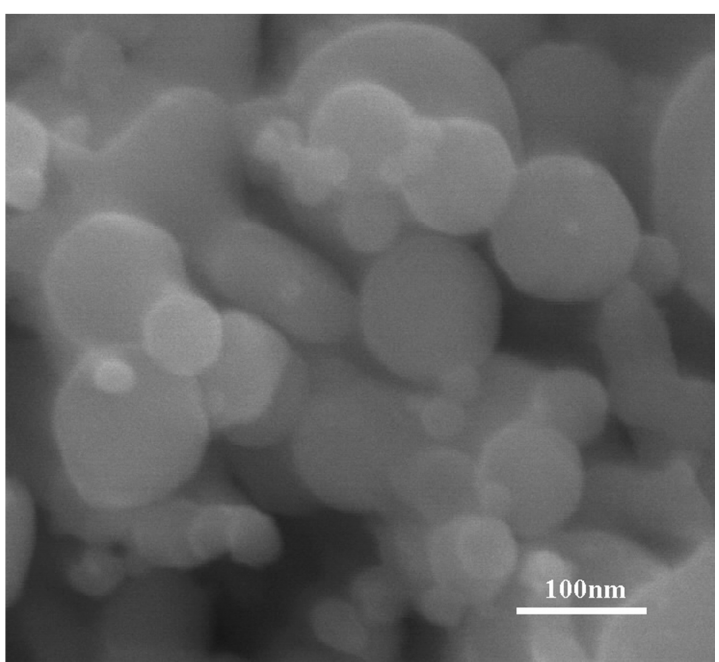

图3 Co纳米粉的扫描电镜 (SEM) 照片。

图2. 是所合成的 $\mathrm{Fe}_{3} \mathrm{O}_{4}$ 空心球的SEM图片及能谱图。从 图2（a）可以观察到明显的厚度较薄的空心结构, 粉体颗 粒均匀, 大小约 $200 \mathrm{~nm}$ 。图2（b) 是箭头所指部位的能谱

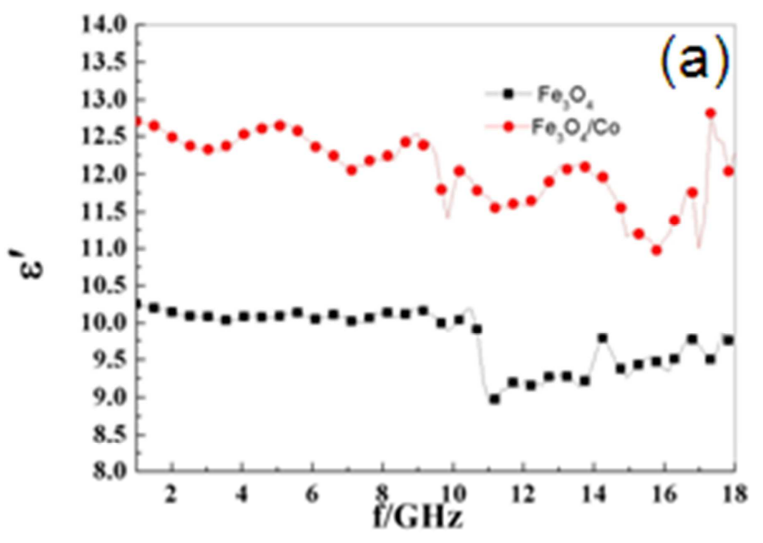

曲线, 从能谱图中可以看到，仅有 $\mathrm{Fe} 、 0$ 两种元素, $\mathrm{Fe}_{3} \mathrm{O}_{4}$ 空心球纯度高, 不包含其他杂质。

图3. 是制备的Co纳米粉的SEM图像, 图像充分显示了 电弧法制备的Co纳米粉呈球形, 尺寸大约为 $100 \mathrm{~nm}$, 由于 磁相互作用使颗粒铰链在一起。

图4. 是 $\mathrm{Fe}_{3} \mathrm{O}_{4} / \mathrm{Co}$ 复合吸波材料复介电常数与频率关系 曲线图,（a）图可以看到在 $2-18 \mathrm{GHz}$ 频率范围内, $\mathrm{Fe}_{3} \mathrm{O}_{4} / \mathrm{Co}$ 复合吸波材料复介电常数实部 $\boldsymbol{E}^{\prime}$ 数值比单纯的 $\mathrm{Fe}_{3} \mathrm{O}_{4}$ 吸波材 料数值大, 表明 $\mathrm{Fe}_{3} \mathrm{O}_{4} / \mathrm{Co}$ 复合吸波材料储存的电场能量多。

（b）图中复介电常数虚部 $\mathcal{E}^{\prime \prime}$ 表示材料对电磁波的损耗性 能, 从整体来看 $\mathrm{Fe}_{3} \mathrm{O}_{4} / \mathrm{Co}$ 复合吸波材料磁损耗比 $\mathrm{Fe}_{3} \mathrm{O}_{4}$ 多, 这 是由于界面损耗和弛豫效应引起的。在2-18 GHz频率范围 内 $\mathrm{Fe}_{3} \mathrm{O}_{4} / \mathrm{Co}$ 复合吸波材料复介电常数虚部出现了多个峰值, 引起这些峰值出现可能是由于Co纳米粒子能级量子化使得 纳米粒子的电子能级发生分离, 分离的能级正处于微波能 量范围。另外晶格电场的热运动下所引起的电子散射、杂 质和由缺陷引起的电子散射、电子与电子之间的作用等都 是产生多个吸收峰的原因。但在 $9-13 \mathrm{GHz}$ 频率范围内 $\mathrm{Fe}_{3} \mathrm{O}_{4}$ 材料的 $\mathcal{E}^{\prime \prime}$ 值大于 $\mathrm{Fe}_{3} \mathrm{O}_{4} / \mathrm{Co}$ 复合材料。

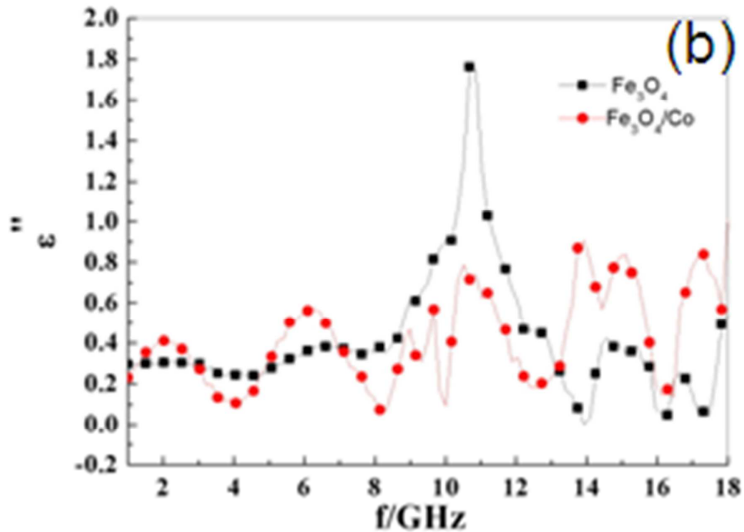

图4 $\mathrm{Fe}_{3} \mathrm{O}_{4} / \mathrm{Co}$ 复合吸波材料复介电常数与频率关系曲线。
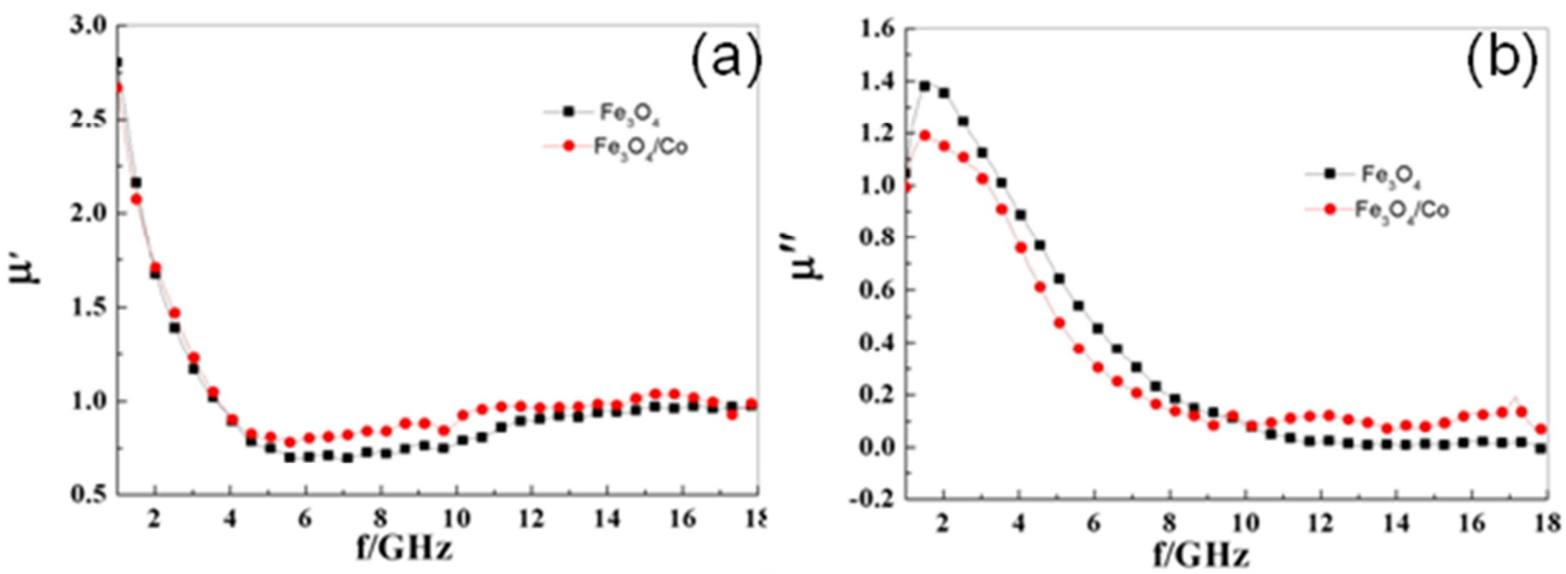

图5 $\mathrm{Fe}_{3} \mathrm{O}_{4} / \mathrm{Co}$ 复合吸波材料复磁导率与频率关系曲线。

图5. 为 $\mathrm{Fe}_{3} \mathrm{O}_{4} / \mathrm{Co}$ 复合吸波材料复磁导率与频率关系 曲线所示在2-18 GHz频段，（a）图中Fe304/Co复合吸波 材料复磁导率实部 $\mu^{\prime}$ 数值变化趋势与 $\mathrm{Fe} 304$ 吸波材料变
化趋势相同, 但数值略大于Fe304吸波材料, 说明其储存 磁场能量大于单一 Fe304吸波材料。（b）图中复磁导率虚 部 $\mu^{\prime \prime}$ 数值都是在 $2-3 \mathrm{GHz}$ 频率范围内先出现最大值后再 
减小的趋势，没有太大区别。一般情况下，吸波材料的吸 波性能并不只由介电常数和磁导率的实部虚部数值决定,

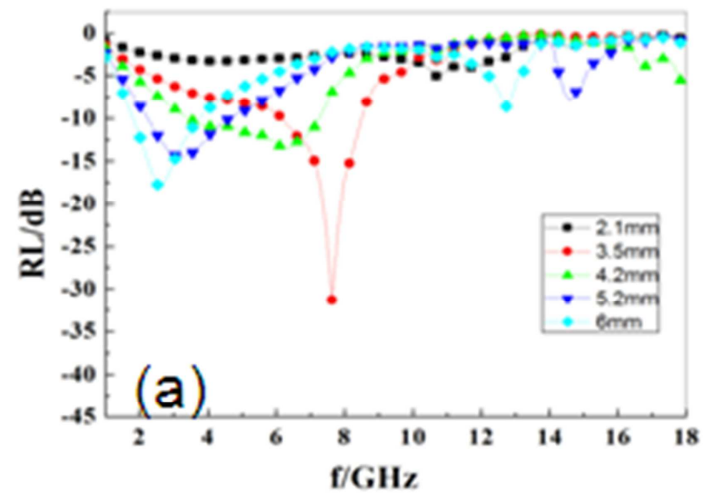

还要看各个电磁参数的匹配。通过改变电磁参数的匹配来 寻找质量轻, 吸收强的性能良好的电磁波吸收剂。

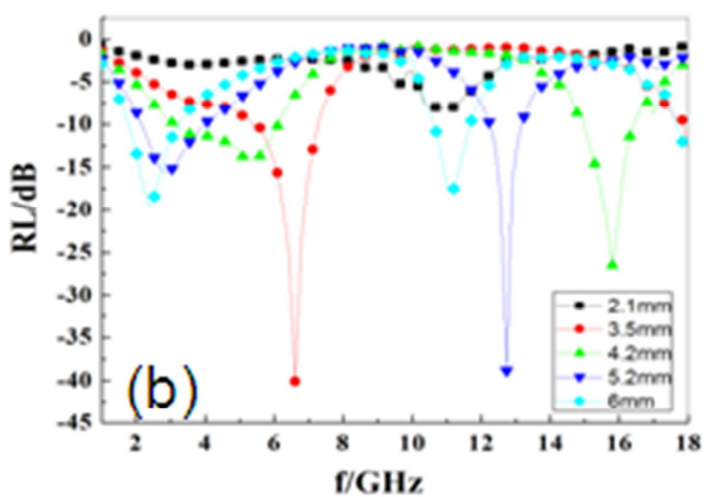

图6 $\mathrm{Fe}_{3} \mathrm{O}_{4} 、 \mathrm{Fe}_{3} \mathrm{O}_{4} / \mathrm{Co}$ 复合吸波材料反射率与频率关系曲线。

图6. 为 $\mathrm{Fe}_{3} \mathrm{O}_{4}$ 与 $\mathrm{Fe}_{3} \mathrm{O}_{4} \mathrm{Co}$ 复合吸波材料反射率与频率关 系曲线图。（a）图为2.1-6 mm空心 $\mathrm{Fe}_{3} \mathrm{O}_{4}$ 吸波材料的模拟 电磁波反射率与频率关系曲线关系。从图上可知, 当涂层 厚度为 $3.5 \mathrm{~mm}$ 时, 在 $7.63 \mathrm{GHz}$ 频率达到 $-31.3 \mathrm{~dB}$ 。当反射损 耗 $<-10 \mathrm{~dB}$ ，对应的频带范围为 $1.85-8.48 \mathrm{GHz}$ 。(b) 图为 为 2. 1-6 mm Fe $\mathrm{O}_{4} / \mathrm{Co}$ 复合吸波材料的模拟电磁波反射率与 频率关系曲线关系。 $\mathrm{Fe}_{3} \mathrm{O}_{4} / \mathrm{C}$ 复合吸波材料在涂层厚度为 $3.5 \mathrm{~mm}$ 时, 材料的吸波能力尤其突出, 频率为 $6.61 \mathrm{GHz}$ 时具 有很大的微波反射损耗值为 $-40.1 \mathrm{~dB}$ 。复合吸波材料在不 同涂层厚度处对应着不同的吸收主峰，当涂层厚度为 4.2 $\mathrm{mm} 、 5.2$ 和 $6 \mathrm{~mm}$ 时，电磁波在 $2-18 \mathrm{GHz}$ 频率范围内出现了 两个吸收峰, 当反射损耗 $<-10 \mathrm{~dB}$, 厚度为 $2.1-6 \mathrm{~mm}$, 对应 $\mathrm{Fe}_{3} \mathrm{O}_{4} / \mathrm{Co}$ 复合吸收剂的吸收频带范围为 $1.85-18 \mathrm{GHz}$, 满足 雷达波吸收材料的要求。

\section{4. 结论}

(1) 水热法制备了空球 $\mathrm{Fe}_{3} \mathrm{O}_{4}, \mathrm{Co}$ 纳米粉和空球 $\mathrm{Fe}_{3} \mathrm{O}_{4} / \mathrm{Co}$ 混合制备微纳米复合吸波材料, $\mathrm{x}$ 射线衍射结构表明 $\mathrm{Fe}_{3} \mathrm{O}_{4}$ 的为面心立方结构, SEM研究揭示所制备的 $\mathrm{Fe}_{3} \mathrm{O}_{4}$ 为空心球, 尺寸约为 $200 \mathrm{~nm}$, 采用的Co纳米粉粒径大约为 $100 \mathrm{~nm}$ 。

(2) 通过将空球 $\mathrm{Fe}_{3} \mathrm{O}_{4}$ 与 $\mathrm{Co}$ 纳米粉进行质量比为 $4: 1$ 的 混合, 吸波剂与石蜡质量比为7: 3 的混合做成吸波样品, 微波吸收性能研究表明, 当吸收层厚度为 $3.5 \mathrm{~mm}$ 时, $\mathrm{Fe}_{3} \mathrm{O}_{4} / \mathrm{Co}$ 复合吸波剂的最佳反射损耗为 $-40.1 \mathrm{~dB}$, 大于同样厚度时 $\mathrm{Fe}_{3} 0_{4}$ 吸收剂的反射损耗 $-31.3 \mathrm{~dB}$ 。当反射损耗 $<-10 \mathrm{~dB}$, 厚度 为 $2.1-6 \mathrm{~mm}$, 对应 $\mathrm{Fe}_{3} \mathrm{O}_{4} / \mathrm{Co}$ 复合吸收剂的吸收频带范围 1. 85- $18 \mathrm{GHz}$, 而对应单一 $\mathrm{Fe}_{3} \mathrm{O}_{4}$ 吸收剂的频带范围为 1. 85-8. 48GHz。研究表明, Co 纳米粉的加入, 有效地调节 了 $\mathrm{Fe}_{3} \mathrm{O}_{4}$ 吸收剂介电损耗和磁损耗的协同作用, 空球 $\mathrm{Fe}_{3} \mathrm{O}_{4} / \mathrm{Co}$ 纳米复合吸波材料明显改善了 $\mathrm{Fe}_{3} \mathrm{O}_{4}$ 纳米吸波材料性能，因 此 $\mathrm{Fe}_{3} \mathrm{O}_{4} / \mathrm{Co}$ 是一种具有潜在实际应用价值的微波吸收剂。

\section{致谢}

本文为辽宁省科技计划项目 (2012222010) 的阶段性 成果之一。

\section{参考文献}

[1] 张淑琴, 张彭. 电磁辐射的危害与防护 [J]. 工业安全与环保, $2008,34(3): 30-37$ 。

[2] 廖少涁. 铁磁学 (下册) [M]. 北京: 科学出版社, 1988:41-48。

[3] H. S. Pan, X. Q. Cheng, C. H. Zhang, et al. Preparation of $\mathrm{Fe}_{2} \mathrm{Ni}_{2} \mathrm{~N} / \mathrm{SiO}_{2}$ Nanocomposite Via A Two-step Route and Investigation of Its Electromagnetic Properties[J].Applied Physics Letters , 2013 , 102 (1) : 012410-012414.

[4] Bregar V B, Jozef S I, Ljubl jana U, et al. Advantages of Ferromagnetic Nanoparticle Composites in Microwave Absorbers [J]. IEEE Transaction on Magnetics, 2004, $36(3): 1679-1684$.

[5] 黄可龙, 孔德明等. 吸波材料的物理机制及其设计 [J]. 中南 工业大学, 2001, 32 (6) :617-621。

[6] Li Y, Chena G, Li Q, et al. Facile Synthesis, Magnetic and Microwave Absorption Properties of $\mathrm{Fe}_{3} \mathrm{O}_{4}$ /polypyrrole core/shell Nanocomposite[J]. Journal of Alloys and Compounds, 2011, 509(10):4104- 4107.

[7] Gómez P H, Munoz J M, Valente M A. Field-Induced Microwave Absorption in Ni Ferrite Nanoparticles[J]. IEEE Transactions Magnetics, 2010, $46(2): 475-478$.

[8] Kong I, Ahmada S H, Abdullah M H, et al. Magnetic and Microwave Absorbing Properties of Magnetite-thermoplastic Natural Rubber Nanocomposites[J]. Journal of Magnetism and Magnetic Materials, 2010, $322(21): 3401-3409$.

[9] 邱琴, 张晏清, 张雄. 电磁吸波材料研究进展 $[\mathrm{J}]$. 电子元件 与材料, $2009,28(8): 78-81$ 。 
[10] Aviles M 0, Chen H T, Ebner A D, et al. In Vitro Study of Ferromagnetic Stents for Implant Assisted-Magnetic Drug Targeting[J].Magnetics Materials , 2007 , $311(1): 306-311$.

[11] Zhang L, Wang T, Liu P, Superparamagnetic Sandwich Fe304@PS@PANiMicrospheres and Yolk/She11 Fe304@PANi Hollow Microspheres With Fe304@PS Nanoparticles As "Partially Sacrificial Templates" [J]. Chemical engineering journal, 2012, 187:372-379.

[12] Ting Lu, Junhu Wang, Jie Yin, et al. Surfactant Effects on The Microstructures of $\mathrm{Fe}_{3} \mathrm{O}_{4}$ nanoparticles synthesized by microemulsion method[J]. Colloids and Surfaces A:Physicochemical and Engineering Aspects, 2013, 436 (5) :675-683.
[13] 0. M. Lemine, K. Omri, B. Zhang, et al. Sol-gel Synthesis of $8 \mathrm{~nm}$ Magnetite $\left(\mathrm{Fe}_{3} \mathrm{O}_{4}\right)$ Nanoparticles and Their Magnetic Properties[J].Superlattices and Microstructures, 2012, 52(4):793-799.

[14] 徐劲峰, 郭方方, 徐政. 六角晶系钡铁氧体纳米晶的制备和 表征 [J]. 同济大学学报, 2004, 32(7):929-932。

[15] 王风龙. $\mathrm{Fe}_{3} \mathrm{O}_{4}$ 空心纳米结构及其复合材料的合成与吸波性 能研究 [D]. 山东大学, 2012。

[16] 张雪峰, 李哲男, 王威娜, 董星龙. 磁性Fe、Co、Ni纳米粒 子的吸波性能研究 [J]. 粉末冶金工业, 2006, 16 (1) : 1116 。 